\title{
Relationship of apical domes in the rabbit uterine epithelium during the peri-implantation period to endocytosis, apocrine secretion and fixation
}

\author{
Margaret B. Parr† and E. L. Parr*† \\ Human Morphology Unit, School of Medicine, The Flinders University of South Australia, Bedford \\ Park, South Australia 5042 and *Department of Anatomy and Histology School of Medicine, The \\ University of Adelaide, Adelaide, South Australia 5000
}

\begin{abstract}
Summary. The luminal surfaces of non-ciliated uterine epithelial cells of 17 rabbits on Days 4-6 of pregnancy were studied histologically with light microscopy, transmission and scanning electron microscopy. The luminal surfaces of most epithelial cells exhibited short microvilli and did not project into the uterine lumen. However, the surfaces of some cells showed fewer microvilli and bulged out into the uterine lumen, giving the cells a dome-like appearance. The domes appeared most frequently on Day 6 of pregnancy and contained a few ribosomes, mitochondria, and membranous elements; large vacuoles and granules were absent.

The domes were not involved in endocytosis. Ferritin introduced into the uterine lumen was incorporated into the cells by coated micropinocytotic invaginations at the base of the microvilli, rather than by any activity of the domes. There was also no indication that the domes pinched off to produce an apocrine secretion. The size and frequency of domes were, however, clearly related to the fixation procedure. Epithelial cells from uteri fixed by vascular perfusion showed fewer and less prominent domes, while cells from uteri fixed by immersion displayed a larger number of domes that projected further into the uterine lumen. The domes in the rabbit epithelium therefore differ structurally and functionally from the apical projections (pinopods) that occur in the uterine epithelium of the rat and mouse during the peri-implantation period.
\end{abstract}

\section{Introduction}

The rabbit endometrium differentiates markedly during early pregnancy (Larsen, 1962; Meyer, 1970; Davies \& Hoffman, 1973; 1975). During the "secretory phase" between Days 4 and 6 of pregnancy, the luminal epithelial cells exhibit characteristic protrusions of the cytoplasm into the uterine lumen in the the form of dome-like prominences (Meyer, 1970; Beier \& Kühnel, 1973; Davies \& Hoffman, 1973, 1975; Beier, 1974; Barberini, Sartori, Motta \& Van Blerkom, 1978). The dome-like projections, often called secretory domes, appeared to be attached to the cells or free within the uterine lumen: it has been suggested that they pinch off from the cells, degenerate, and release their contents into the uterine lumen. The domes were therefore considered to be indicative of an intense apocrine secretory process and related to the synthesis of uterine-specific proteins released into the uterine lumen at this time (Beier, 1974; Barberini et al., 1978). A pical protrusions

$\dagger$ Present address: Department of Anatomy, School of Medicine, Southern Illinois University, Carbondale, Illinois 62901 , U.S.A. 
have also been observed in the uterine epithelial cells of the human, rat and mouse during the progestational period and most investigators have interpreted them as signs of apocrine secretion (see references in Parr \& Parr, 1974).

However, the hypothesis of apocrine secretion in the pregnant uterus is open to question. In the rat and mouse uterine epithelium, apical protrusions appear during the peri-implantation period and are involved in the process of endocytosis rather than apocrine secretion (Enders \& Nelson, 1973; Parr \& Parr, 1974, 1977). Several histological studies of the human uterus did not reveal secretory domes or the pinching off of any part of the apical cell surfaces (Gompel, 1962; Cavazos, Green, Hall \& Lucas, 1967; Sengel \& Stoebner, 1970; Johannisson \& Nilsson, 1972). In the rabbit uterus, Davies \& Hoffman (1975) reported that although cytoplasmic bulging of the cells was truly part of the progestational response and was temporally associated with uterine secretory activity, their observations did not support an intensive secretory role, apocrine or otherwise, for the domeshaped surface cells.

The hypothesis of apocrine secretion by luminal epithelial cells conflicts with studies which have shown that cellular materials are toxic for preimplantation embryos (Parr, 1969; Joshi \& Kraemer, 1970; Smith, El Sahwi, Wilson \& Moyer, 1971). In addition, studies on the luminal epithelium of the epididymis, where apocrine secretion was previously thought to occur, have shown that the apical protrusions of the cells were artefacts of poor fixation (Hamilton, 1972).

In the present investigation we have studied the secretory domes or apical protusions in the nonciliated luminal epithelial cells of the rabbit uterus during the peri-implantation period to determine whether the domes were related to endocytosis, apocrine secretion, or fixation procedures.

\section{Materials and Methods}

Rabbits of mixed breeds were obtained from Adelaide University Animal House. The animals were mated and the day of mating was designated Day 0 . Ovulation was verified by histological examination of ovaries for the presence of corpora lutea. On Days 4, 5, and 6 of pregnancy, 11 rabbits were anaesthetized with pentobarbitone sodium and ether, and 0.25 or $0.50 \mathrm{ml}$ of a $5 \%(\mathrm{w} / \mathrm{v})$ solution of ferritin (Nutritional Biochemicals Corp., Cleveland; purified by centrifugation, Parr \& Oei, 1973) in medium 199 was injected slowly into the uterine lumen at the ovarian end of the right horns. Approximately $1 \mathrm{~h}$ later the rabbit uteri were fixed by vascular perfusion with a mixture of $2.5 \%$ glutaraldehyde and $2 \%$ paraformaldehyde in $0 \cdot 1 \mathrm{M}$-cacodylate, $\mathrm{pH} 7 \cdot 4$, or they were removed from the animals, cut into small segments and fixed by immersion in the same fixative. In one rabbit, the control uterine horn (not injected with ferritin) was fixed approximately 10 min post

\section{PLATE 1}

Fig. 1. A transmission electron micrograph of a rabbit uterus, fixed by vascular perfusion on Day 5 of pregnancy, shows luminal epithelial cells and ferritin $(F)$ in the uterine lumen (L). The luminal surfaces of the epithelial cells are flat or dome-shaped (D) and covered with short microvilli. The supranuclear cytoplasm contains ribosomes, rough endoplasmic reticulum, numerous elongated mitochondria, Golgi complex (G), and a few lysosomes (LY). Note the absence of secretory granules or large vacuoles in the cells. $\times 7900$.

Fig. 2. A transmission electron micrograph of a rabbit uterus fixed by immersion fixation 10 min after death shows the apical portions (domes) of some epithelial cells projecting into the uterine lumen. The domes are covered with a few short microvilli and contain ribosomes, membranous elements, mitochondria and a centriole. One of the domes has a stalk-like base continuous with the apical part of the cell. Small spherical masses of cytoplasm which appear to be lying free in the lumen close to the dome may well be attached to the dome at another plane of section. They contain cytoplasm with the same electron density as in intact cells, indicating that they are not releasing their contents. Note the absence of secretory granules and vacuoles in the domes. $\times 19800$. 


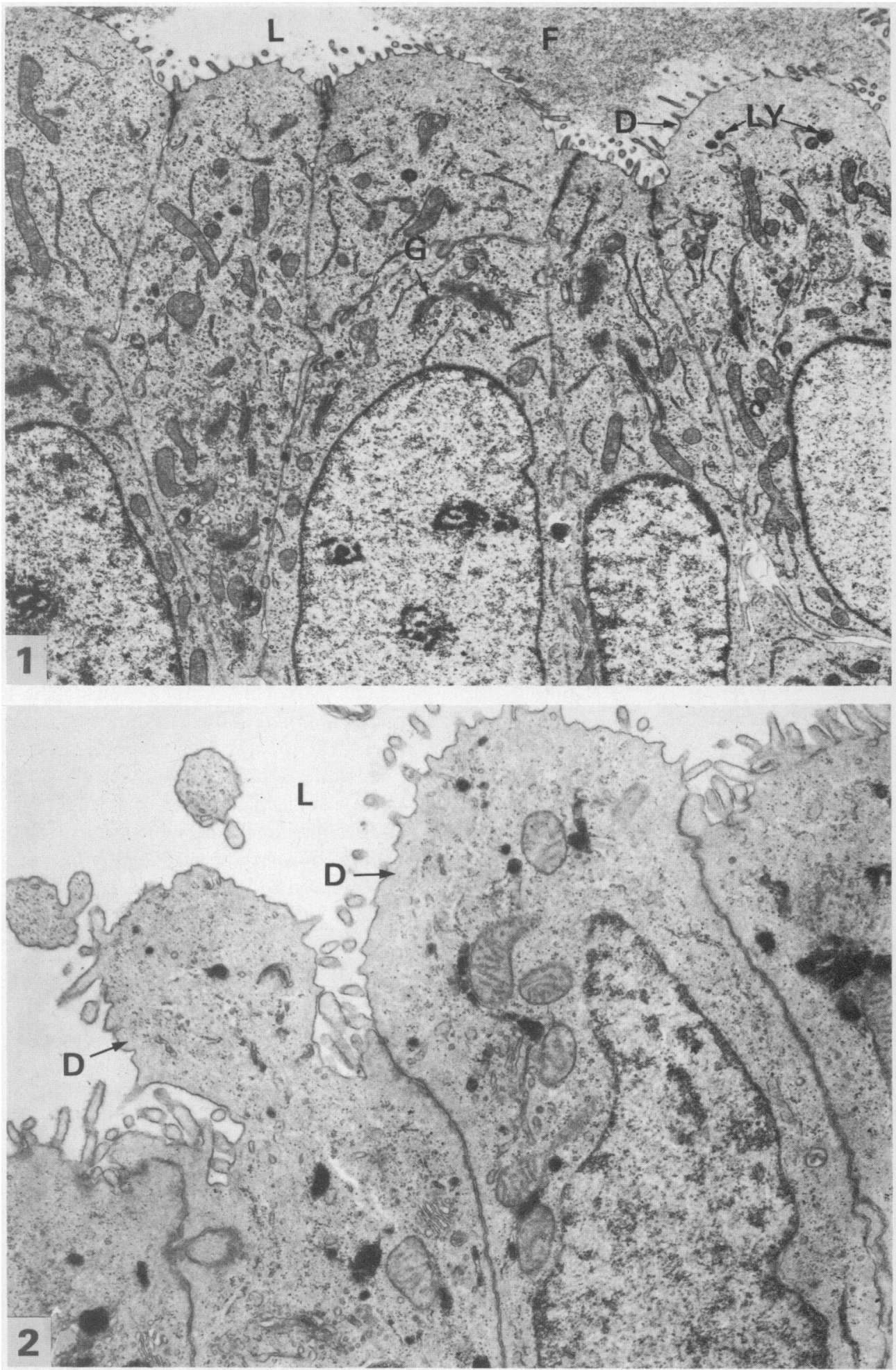

(Facing p. 740) 


\section{PLATE 2}

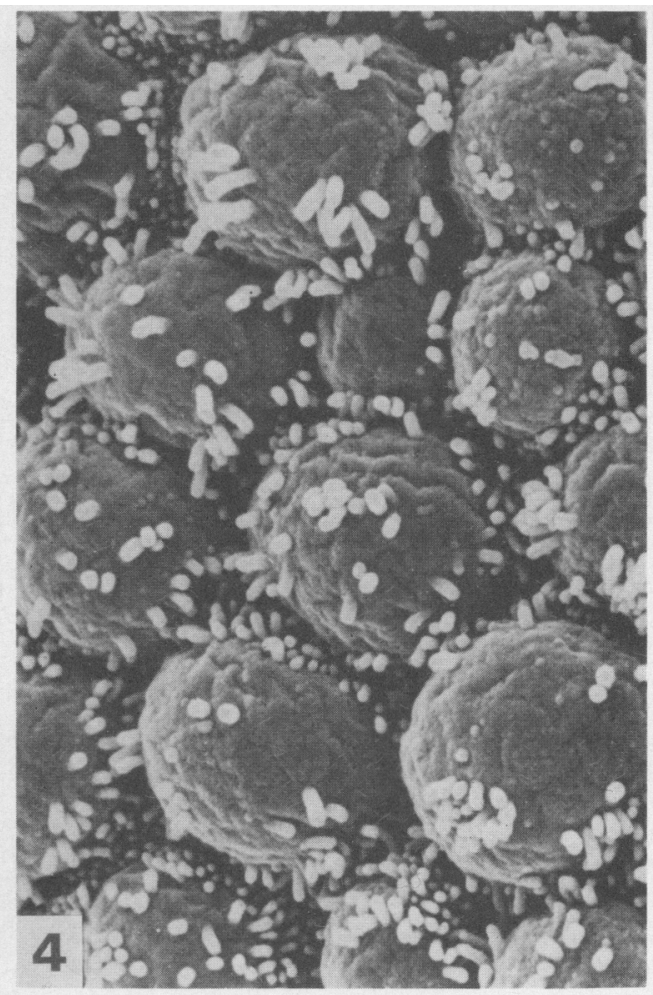

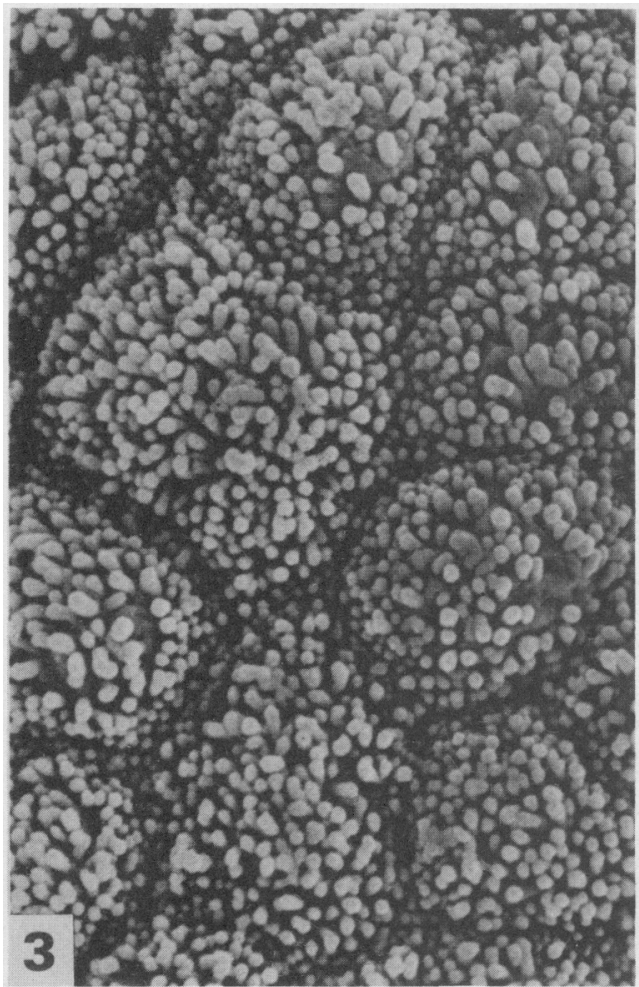

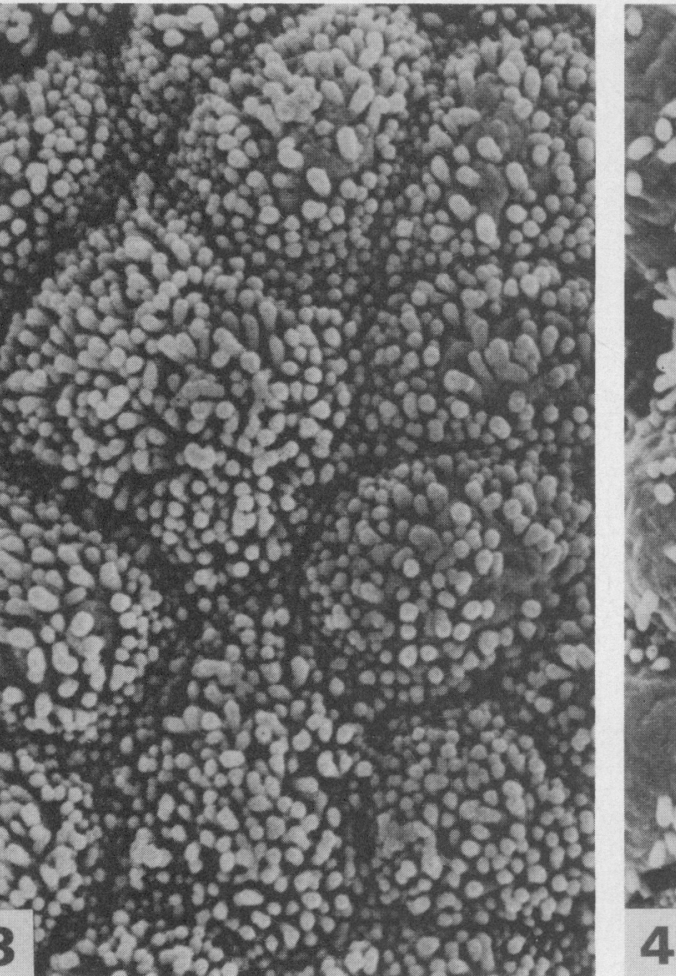

\section{8}

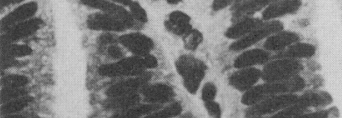

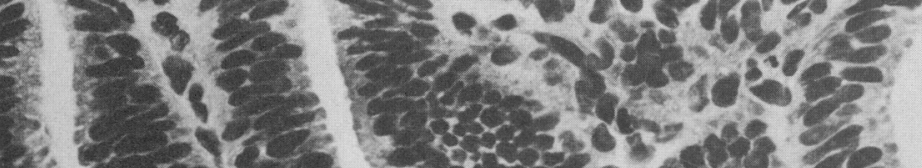

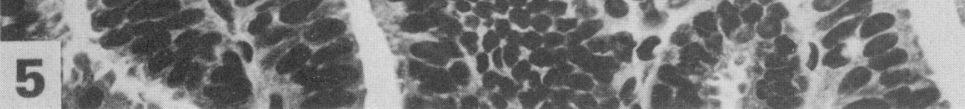

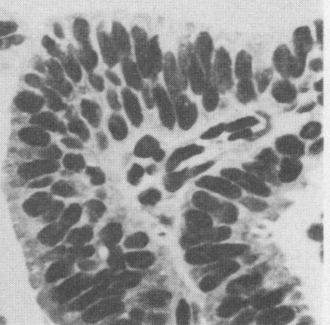

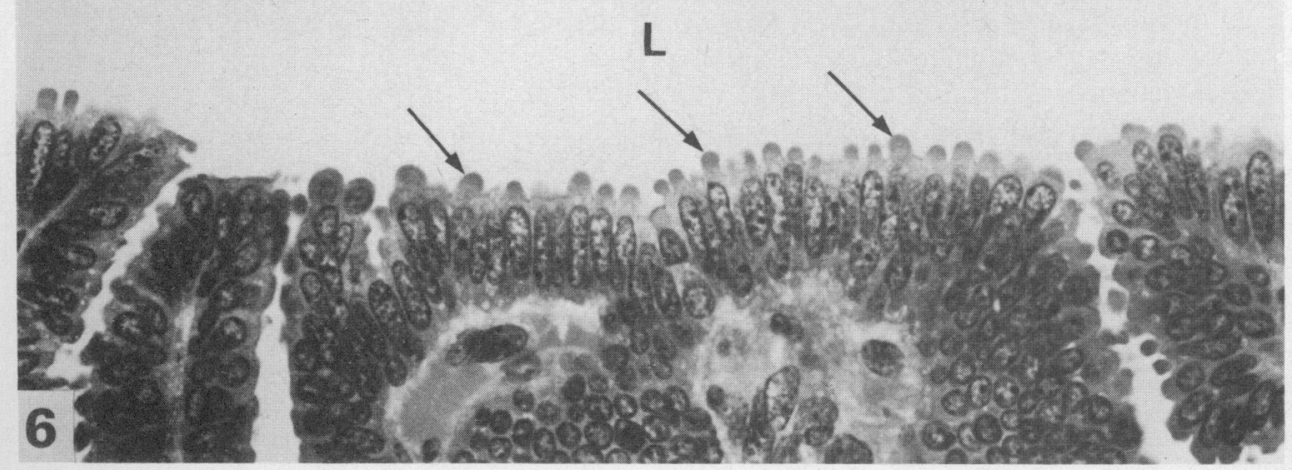


mortem. Small uterine segments from both groups of rabbits were then dehydrated and embedded in paraffin wax, and sections $(5 \mu \mathrm{m})$ were stained with Perls' stain (Pearse, 1972) for the demonstration of iron. In addition, uterine tissues from both horns were processed for transmission electron microscopy. Thin uterine segments were washed in cacodylate buffer overnight, post-fixed for $1 \mathrm{~h}$ $\left(4^{\circ} \mathrm{C}\right)$ in a mixture of $1 \%$ osmium tetroxide and $1.5 \%$ potassium ferrocyanide in distilled water (Karnovsky, 1971), washed, dehydrated and, embedded in Spurr's medium. Semi-thin sections were stained with toluidine blue in borate buffer. Thin sections were stained with aqueous uranyl acetate and lead citrate and examined in a Siemens Elmiskop 102 electron microscope.

The uteri of 6 additional rabbits on Days 5 and 6 of pregnancy were ligated before removal from the animals in an attempt to trap any material secreted into the uterine lumen and prevent its loss during processing. The ligatures were placed after fixation in 3 of these rabbits whose uteri were fixed by vascular perfusion, and before fixation in the other 3 animals whose uteri were fixed by immersion. The uterine horns were removed and the $1-2 \mathrm{~cm}$ ligated segments were immersion fixed for $24 \mathrm{~h}$, rinsed in buffer, and dehydrated. The ligated portions at the ends of the uterine pieces were cut off just before embedding to allow more complete infiltration of the embedding medium and the whole central portions were embedded intact in glycol methacrylate (Polysciences, Inc.). After the resin had polymerized, each block was cut in half (cross-section) and $2 \mu \mathrm{m}$ sections of uterine tissue from the centre of the block were cut and stained with haematoxylin and eosin. Thin segments of the contralateral non-ligated horns were also processed in glycol methacrylate, sectioned and stained. Segments of immersion- or perfusion-fixed non-ligated horns from these 6 rabbits were also cut open to expose the luminal surfaces and were then processed for scanning electron microscopy. The segments were washed, post-fixed in $2 \%$ osmium tetroxide $\left(1 \mathrm{~h}, 4^{\circ} \mathrm{C}\right)$, washed, dehydrated, and dried by the critical-point method. The dried uterine tissues were then mounted on stubs, gold coated, and examined with an ETEC Autoscan scanning electron microscope.

\section{Results}

Histological appearance of domes. The majority of the non-ciliated epithelial cells of rabbit uteri fixed by vascular perfusion on Days 4, 5 and 6 of pregnancy exhibited luminal surfaces that were covered by short microvilli and which did not project into the uterine lumen. However, the surface of some cells showed fewer microvilli and bulged out into the uterine lumen to give the cells a domelike appearance (PI. 1, Fig. 1). The domes often involved the entire luminal surface of the cells, but in some uterine cells fixed by immersion the projections extended from only part of the surface, and were attached to the rest of the cell by means of narrower stalks of cytoplasm (Pl. 1, Fig. 2). The

\section{PLATE 2}

Fig. 3. A scanning electron micrograph of the rabbit uterus on Day 6 of pregnancy, fixed by vascular perfusion, shows the luminal surface of the uterine epithelial cells covered by numerous short closely packed microvilli. The apical portions of the cells project slightly into the uterine lumen causing the borders of the cells to appear recessed. $\times 6700$.

Fig. 4. A scanning electron micrograph of the rabbit uterus on Day 6 of pregnancy, fixed by immersion fixation, shows the luminal surface of the uterine epithelial cells covered by few, short, widely-spaced microvilli. The apical portions of the cells project markedly into the uterine lumen causing the borders of the cells to appear deeply recessed. $\times 6600$.

Fig. 5. Light micrograph of a rabbit uterus on Day 5 of pregnancy fixed by vascular perfusion. Note the paucity of apical domes. The apical surface of most cells appears flattened. $\mathrm{L}=$ lumen. $\times 350$.

Fig. 6. Light micrograph of a rabbit uterus on Day 6 of pregnancy fixed by immersion. Many conspicuous domes (arrows) appear in the epithelium. $\mathrm{L}=$ lumen. $\times 550$. 
dome-like protrusions were not an all-or-nothing phenomenon, but showed the complete range of size or development from very slight apical bulging to quite obvious bulbous projections. Generally, the cytoplasm in the obvious domes contained a few ribosomes, mitochondria, and membranous elements, but large vacuoles and granules were absent; occasionally, a part of the nucleus extended into the dome. At least a few cells showing domes were present in all uterine tissues examined but the domes appeared more frequently in uteri of rabbits on Day 6 of pregnancy.

Domes and endocytosis. The domes were not related to endocytosis. After intraluminal administration of tracer ferritin on Days 4, 5 and 6 of pregnancy, the Perls' stain for iron showed ferritin in the uterine and glandular lumen but not in the epithelial cells. Ultrastructural observations revealed ferritin in a few coated micropinocytotic invaginations and vesicles, and in very small amounts in a few lysosome-like organelles in the apical cytoplasm, but not in relation to domes. Pinocytosis did not appear to be a prominent epithelial cell activity and most epithelial cells failed to show any ferritin incorporation at all. There were few lysosome-like organelles, such as multivesicular bodies and dense bodies, present in the cytoplasm and the Golgi apparatus was relatively small and consisted of stacks of short flattened cisternae. There was an absence of pinopod-like structures projecting from the apical surface and there were no large endocytotic vacuoles in the domes or in the apical cytoplasm.

Domes and apocrine secretion. When the domes appeared in the uterine cells they were usually connected to the apical parts of the cells and did not appear to be releasing their contents into the uterine lumen. Spheres of cytoplasm resembling the domes were occasionally seen free in the lumen, but they could have been connected to cells at another plane of section (P1. 1, Fig. 2). All domes, whether connected to cells or not, exhibited the same uniformly dense cytoplasmic staining as did cytoplasm in the remainder of the cells. If domes were releasing their cytoplasm and other contents into the uterine lumen a variable staining density in the domes would be expected. There was no morphological indication of secreted material in the uterine lumen, such as membranelimited vesicles or membranous debris. Such material could have been washed out of the uterine lumina during the histological preparation of the tissues, but this seems unlikely because tracer ferritin was readily identified in lumina of tissues prepared in similar ways (P1. 1, Fig. 1). Scanning electron micrographs of the luminal surface showed domes of various heights, but there were no spheroidal elements that appeared to be unattached to the underlying cells (Pl. 2, Figs $3 \& 4$ ). Furthermore, there was no sign of secreted material in the lumina of 5 out of 6 uteri that were ligated before processing to prevent luminal material from being washed out. In one uterus processed in this way, some material possibly resembling secreted material was present in the lumen. This uterus, which was fixed by immersion rather than vascular perfusion, showed irregularities in the surface of the epithelium that were probably due to poor fixation.

Domes and tissue fixation. Domes were observed in at least some uterine cells in all preparations but their incidence was clearly related to tissue fixation. Cells in 10 uteri fixed by vascular perfusion showed fewer and less prominent domes (P1. 2, Figs $3 \& 5$ ) while cells in 6 uteri fixed by immersion displayed a greater number of domes which projected further into the lumen (P1. 2, Figs 4 \& 6).

\section{Discussion}

The domes that we observed at the luminal surface of rabbit uterine epithelial cells on Days 4-6 of pregnancy were similar to those reported by Meyer (1970), Beier \& Kühnel (1973), Davies \& Hoffman $(1973,1975)$ and Beier (1974). Although the apical protrusions of the rabbit uterine epithelium on Days 4-6 of pregancy have been likened to the protrusions (pinopods) observed in rat and mouse uteri at comparable times (Martin, 1980), the present results indicate that they differ morphologically and functionally from those structures. In rat and mouse uteri the pinopods showed irregular surfaces with many folds and invaginations devoid of microvilli, and they often appeared as cup-shaped projections. They contained filamentous cytoplasm devoid of other organ- 
elles and a large vacuole was usually present. The pinopods were shown to be vehicles for endocytosis since intraluminal tracers, such as ferritin and horseradish peroxidase, were found inside the cups, in the large vacuoles inside the apical projections, and subsequently in smaller vacuoles and lysosomes deeper in the cytoplasm (Enders \& Nelson, 1973; Parr \& Parr, 1974, 1977). The domes that occurred in the rabbit epithelium differed in many morphological aspects from the pinopods found in rodent uteri, did not contain vacuoles, and were not involved in endocytosis because intraluminal ferritin did not associate with the domes in any way.

Our observations also do not support the involvement of apical domes in apocrine secretion. We were unable to demonstrate any morphological correlate of secreted material, such as membrane debris, in the uterine lumen with or without ligation of the uterine horns, and there were no morphological indications that apical domes released their contents because the density of cytoplasmic staining appeared uniform in all domes.

The apical domes were, at least to some extent, a fixation artefact. The dome formations were larger and more numerous in uterine tissues fixed by immersion, as compared to those fixed by vascular perfusion. The correlation between fixation procedure and appearance of domes is supported by previous observations. Immersion-fixed tissues showed numerous prominent apical protrusions (Meyer, 1970; Barberini et al., 1978) while perfusion-fixed epithelial cells displayed shallow domes (Davies \& Hoffman, 1975). Beier \& Kühnel (1973) reported numerous apical protrusions in cells fixed by perfusion, but the fixative used $(5 \%$ glutaraldehyde) was ice-cold and the ultrastructure of the epithelial cells showed obvious signs of poor fixation, such as swollen Golgi vesicles and enlarged vacuoles. It remains uncertain whether any additional improvements in the fixation procedure to provide better preservation of normal cell morphology would reduce the number of domes still further. Our observations, however, emphasize the need for careful preparation of rabbit uterine tissues to avoid the development of morphological artefacts due to inadequate fixation and for cautious evaluation of the possible functional significance of cytological features observed in histological preparations.

\section{References}

Barberini, F., Sartori, S., Motta, P. \& Van Blerkom, J. (1978) Changes in the surface morphology of the rabbit endometrium related to the estrous and progestational stages of the reproductive cycle. A scanning and transmission electron microscopic study. Cell Tissue Res. 190, 207-222.

Beier, H.M. (1974) Oviductal and uterine fluids. J. Reprod. Fert. 37, 221-237.

Beier, H.M. \& Kühnel, W. (1973) Pseudopregnancy in the rabbit after stimulation by human chorionic gonadotropin. Horm. Res. 4, 1-27.

Cavazos, F., Green, J.A., Hall, D.G. \& Lucas, F.U. (1967) Ultrastructure of the human endometrial glandular cell during the menstrual cycle. Am. J. Obstet. Gynec. 99, 833-854.

Davies, J. \& Hoffman, L.H. (1973) Studies on the progestational endometrium of the rabbit. I. Light microscopy, day 0 to day 13 of gonadotropin-induced pseudopregnancy. Am. J. Anat. 137, 423-445.

Davies, J. \& Hoffman, L.H. (1975) Studies on the progestational endometrium of the rabbit. II. Electron microscopy, day 0 to 13 of gonadotropin-induced pseudopregnancy. Am. J. Anat. 142, 335-366.

Enders, A.C. \& Nelson, D.M. (1973) Pinocytotic activity of the uterus of the rat. Am. J. Anat. 138, 277-300.

Gompel, C. (1962) Ultrastructure of the human endo- metrial cell studied by electron microscopy. Am. J. Obstet. Gynec. 84, 1000-1009.

Hamilton, D.W. (1972) The mammalian epididymis. In Reproductive Biology, pp. 268-337. Eds H. Balin \& S. Glasser. Excerpta Medica, Amsterdam.

Johannisson, E. \& Nilsson, L. (1972) Scanning electron microscopic study of the human endometrium. Fert. Steril. 23, 613-625.

Joshi, S.G. \& Kraemer, D.C. (1970) Development of mouse embryos in uterine washings of rats and baboons bearing an intrauterine foreign body. Contraception 2, 353-359.

Karnovsky, M.J. (1971) Use of ferrocyanide-reduced osmium tetroxide in electron microscopy. Proc. 11th A. Meeting, Am. Soc. Cell Biol. Abstr. 284.

Larsen, J.F. (1962) Electron microscopy of the uterine epithelium in the rabbit. J. Cell Biol. 14, 49-64.

Martin, L. (1980) What roles are fulfilled by uterine epithelial components in implantation? Prog. Reprod. Biol. 7, 54-69.

Meyer, J.M. (1970) Recherches sur l'ultrastructure de la maqueuse utérine de la lapine. Arch. Anat. Histol. Embryol. 53, 1-40.

Parr, E.L. (1969) Intrauterine foreign bodies: a toxic effect of leukocyte extracts on rat morulae in vitro. Biol. Reprod. 1, 1-10. 
Parr, E.L. \& Oei, J.S. (1973) Immobilization of membrane $\mathrm{H}_{2}$ antigens by paraformaldehyde fixation. $J$. Cell Biol. 59, 537-542.

Parr, M.B. \& Parr, E.L. (1974) Uterine luminal epithelium: protrusions mediate endocytosis, not apocrine secretion, in the rat. Biol. Reprod. 11, 220-233.

Parr, M.B. \& Parr, E.L. (1977) Endocytosis in the uterine epithelium of the mouse. J. Reprod. Fert. 50, 151153.

Pearse, A.G.V. (1972) Histochemistry, Theoretical and Applied, Vol. 2, p. 1402. Churchill Livingstone, Edinburgh.
Sengel, A. \& Stoebner, P. (1970) Ultrastructure de l'endometre humain normal. Les glandes. Z. Zellforsch. mikrosk. Anat. 109, 260-278.

Smith, D.M., El Sahwi, S., Wilson, N. \& Moyer, D.L. (1971) Effect of polymorphonuclear leukocytes on the development of mouse embryos cultured from the two-cell state to blastocysts. Biol. Reprod. 4, 74-83.

Received 4 June 1982 